\title{
Relación entre Satisfacción Laboral, Estrés Laboral y sus Resultados en Trabajadores de una Institución de Beneficencia de la Provincia de Concepción
}

\author{
RELATIONSHIP BETWEEN JOB SATISFACTION, WORK STRESS AND ITS RESULTS IN WORKERS OF A CHARITY \\ INSTITUTION OF THE PROVINCE OF CONCEPCIÓN
}

Maria Margarita Chiang Vega1, Gabriel Rodrigo Riquelme Neira², Pedro Alfonso Rivas Escobar ${ }^{3}$

1. Ingeniero Comercial, Master en Recursos Humanos, Doctora por la U. Pontificia de Madrid.

2. Psicólogo, USS, Diplomado en Coaching y Liderazgo, UNAB.

3. Psicólogo, USS.

\section{RESUMEN}

El objetivo de esta investigación es analizar la relación entre el estrés laboral y la satisfacción laboral en una Institución de Beneficencia en la Provincia de Concepción. Se desarrolla un estudio empírico transversal, descriptivo correlacional, con un universo del estudio conformado por 184 trabajadores, segmentado en cinco grupos. El cuestionario autoadministrado y anónimo con 71 ítems divididos en 3 secciones: información general, estrés laboral y satisfacción laboral. El análisis de fiabilidad del cuestionario presento valores de alfa de Cronbach, para Satisfacción Laboral entre 0,656 y 0,923, para Estrés Laboral entre 0,635 y 0,927 siendo aceptable la fiabilidad en ambos. En general, respecto al estrés todos los grupos de trabajo se ubican en el cuadrante baja tensión, baja demanda psicológica, alto control laboral y alto apoyo global. Respecto a Satisfacción laboral el promedio general en las líneas de trabajo evaluadas es de 3,8 puntos, lo que denota un nivel mediano de satisfacción laboral.

Las correlaciones permiten concluir que a mayor percepción de las variables de Satisfacción laboral y mayor Control sobre el trabajo, se logra reducir el grado de estrés. Además la dimensión de Apoyo Social Laboral Global aumenta la Satisfacción Laboral, permitiendo disminuir el estrés.

(Chiang M, Riquelme G, Rivas P, 2018. Relación entre Satisfacción Laboral, Estrés Laboral y sus Resultados en Trabajadores de una Institución de Beneficencia de la Provincia de Concepción. Cienc Trab. Sep-Dic; 20 [63]: 178-186).

Palabras claves: SATISFACCIÓN LABORAL, ESTRÉS LABORAL.

\section{ABSTRACT}

The objective of this research is to analyze the relationship between work stress and job satisfaction in a Charity Institution in the Province of Concepción. A cross-sectional, descriptive, correlational empirical study was developed, with a study universe consisting of 184 workers, divided into five groups. The self-administered and anonymous questionnaire with 71 items divided into 3 sections: general information, work stress and job satisfaction. The reliability analysis of the questionnaire presented values of Cronbach's alpha, for Labor Satisfaction between 0.656 and 0.923, for Labor Stress between 0.635 and 0.927 , the reliability being acceptable in both. In general, with respect to stress, all work groups are located in the low tension, low psychological demand, high labor control and high global support quadrant. Regarding Job Satisfaction, the general average in the evaluated work lines is 3.8 points, which denotes a medium level of job satisfaction.

The correlations allow us to conclude that the greater the perception of the variables of job satisfaction and greater control over work, the reduction of the degree of stress is achieved. In addition, the dimension of Global Labor Social Support increases the Labor Satisfaction, allowing to reduce stress.

Key words: JOB SATISFACTION, WORK STRESS // WORKS SATISFACTION, WORKS STRESS

\section{INTRODUCCIÓN}

En la época actual y de manera cotidiana podemos visualizar el impacto que se produce en la interacción persona y organización, lo anterior se puede apreciar en su calidad de vida, el clima

Correspondencia / Correspondence:

Gabriel Rodrigo Riquelme Neira

Calle Pinares 24, Casa 84, Condominio Alto Castaños II,

Comuna Chiguayante

Tel.: +56951265217

e-mail: griquelme@hogardecristo.cl, gabrielriq@gmail.com

Recibido: 8 de junio de 2018 / Aceptado: 25 de septiembre de 2018 laboral, satisfacción de los trabajadores, permanencia, compromiso y productividad de éstos, por mencionar algunos. Eso sí, la permanencia en el tiempo de una organización, está en cómo se va reinventando en el espacio y época, de tal manera de no verse afectada su estabilidad y primordialmente su producción.

El objetivo del presente trabajo es buscar la posible relación entre las variables de estrés y satisfacción laboral, comparando trabajadores con tipos de contratos indefinidos y a plazo, en trabajadores de una organización de beneficencia chilena.

\section{Satisfacción Laboral ${ }^{1}$}

En la actualidad no existe una definición unánimemente aceptada sobre el concepto de satisfacción laboral.

A pesar de la cantidad y diversidad de investigaciones llevadas a cabo en torno a la satisfacción en el trabajo, la comprensión de 
sus causas está lejos de clarificarse. Todavía hoy, se intenta comprender dónde residen sus determinantes, si en la naturaleza del trabajo en sí, en las variables del trabajador o en la satisfacción resultante de las interacciones establecidas entre el ciudadano activo (respectiva historia de vida y personalidad) y las particularidades del contexto de trabajo. ${ }^{2}$

Así, la satisfacción laboral es uno de los ámbitos de la calidad de vida laboral que ha captado mayor interés. En un primer momento, la atención se centró en los efectos de la satisfacción laboral sobre variables como la accidentabilidad, el absentismo, el cambio organizacional y el abandono de la organización. ${ }^{3}$

Snyder ${ }^{4}$ indica algunas razones que explican la gran atención dedicada a la satisfacción laboral: 1) La satisfacción en el trabajo es un resultado importante de la vida organizacional. 2) La satisfacción ha aparecido en diferentes investigaciones como un predictor significativo de conductas disfuncionales importantes, como el absentismo, el cambio de puesto y de organización.

Se ha observado que, la satisfacción laboral entendida como un factor que determina el grado de bienestar que un individuo experimenta en su trabajo, se está convirtiendo en un problema central para la investigación de la organización. ${ }^{5}$ Así, es uno de los ámbitos de la calidad de vida laboral que ha captado mayor interés.

Sin embargo, a pesar de su larga tradición en la psicología de las organizaciones, el concepto de satisfacción laboral ha sido definido de múltiples formas, tantas como autores han teorizado sobre él.

Encontrándose una serie de definiciones que hacen referencia a la satisfacción laboral como estado emocional, sentimientos o respuestas afectivas. Locke $^{6}$ lo define como estado emocional positivo o placentero resultante de la percepción subjetiva de las experiencias laborales de la persona. En la misma línea, Mueller y McCloskey ${ }^{7}$, plantean que es una orientación afectiva positiva hacia el empleo. Para Newstrom y Davis ${ }^{8}$, la satisfacción corresponde a un conjunto de sentimientos y emociones favorables o desfavorables con que los empleados ven su trabajo.

Otro grupo de estudiosos, plantean que la satisfacción laboral, no sólo debe hacer énfasis en lo emocional; por ello su grado de relevancia que ésta tiene en el comportamiento laboral; de esta manera, ven a la satisfacción laboral como una actitud general ante el trabajo. En este sentido, Bravo, Peiró \& Rodríguez ${ }^{9}$, la definen como una actitud o conjunto de actitudes desarrolladas por la persona hacia su situación de trabajo, actitudes que pueden ir referidas hacia el trabajo en general o hacia facetas especificas del mismo.

Luego, la satisfacción laboral es, básicamente, un concepto globalizador con el que se hace referencia a las actitudes de las personas hacia diversos aspectos de su trabajo. Por consiguiente, hablar de satisfacción laboral implica hablar de actitudes. ${ }^{9}$

Otra definición importante es la ofrecida por Davis y Newstrom ${ }^{10}$, quienes definen la satisfacción en el trabajo como un conjunto de sentimientos favorables y desfavorables mediante los cuales los empleados perciben su trabajo. La satisfacción laboral es cambiante porque crecen y decrecen los sentimientos satisfactorios a medida que los motivos de logro se van cubriendo, por lo que las intensidades iniciales se complementan a través de las conductas realizadas.

La satisfacción en el trabajo es importante en cualquier tipo de profesión; no sólo en términos del bienestar deseable de las personas donde quiera que trabajen, sino también en términos de productividad y calidad. ${ }^{10}$

\section{Estrés Laboral}

Todos manejamos la palabra estrés y otros términos relacionados, como miedo, ansiedad, angustia, fatiga o depresión, pero es complejo delimitar con claridad el significado de estrés, su importancia y las consecuencias asociadas por quienes lo padecen de manera constante. Así, los términos de estrés, ansiedad y miedo se entremezclan en el lenguaje cotidiano e incluso en el lenguaje científico ${ }^{11}$, de manera que su definición es compleja. Esto provoca que el estudio del estrés haya adquirido importancia y preocupación en la actualidad, por ser la causa de numerosas enfermedades que afectan a la sociedad en general.

El estrés es un fenómeno asociado a múltiples factores y si, bien no puede atribuirse únicamente a las situaciones laboral concreta, esta es una de las principales esferas de actividad de los individuos y uno de los principales contextos a analizar a la hora de estudiar el estrés psicosocial y su relación con la salud. El estrés puede producirse en cualquier lugar de trabajo y afectar a cualquier trabajador, independiente del tamaño de la empresa, de su ámbito de actividad, del tipo de contrato o relación del trabajo. ${ }^{11}$ En la actualidad, un porcentaje significativo de las demandas profesionales que reciben los psicólogos, son aquellas relacionadas con los factores de tipo psicosocial propios del lugar de trabajo; los que originan en los trabajadores que los padecen numerosos problemas en su salud, tanto a nivel físico como psíquico. No debemos olvidar que en toda organización de trabajo existen distintos agentes que pueden afectar la esfera social y psicológica de los trabajadores ${ }^{12}$, son los que se ha dado en llamar riesgos psicosociales y refieren a aspectos de la concepción, organización y gestión del trabajo, así como de su contexto social y ambiental, que tienen la potencialidad de causar daños físicos, sociales o psicológicos en los trabajadores y que en los últimos años se están convirtiendo en uno de los principales problemas de salud laboral. ${ }^{13}$

Lo anterior podría confirmar lo que plantea Bennis ${ }^{14}$, citado por Chandía et $\mathrm{al}^{15}$ para que una organización sea saludable debe ser capaz de mantener un contacto adecuado con su entorno y adaptarse a él, a su vez desarrollar y mantener su identidad como organización e infundir aquello a sus trabajadores, para potenciar y mantener la integridad interna y de establecer una misión, estrategias y expectativas de desarrollo definidas. Al existir un deterioro de la calidad de vida laboral en una organización, los niveles elevados de estrés pueden repercutir negativamente sobre las contribuciones que sus recursos humanos han de realizar para preservar ese estado saludable.

El concepto estrés fue acuñado por Hans Selye en $1936^{16}$, quien lo define como un síntoma general de adaptación. El estrés seria la respuesta general del organismo ante cualquier estimulo estresor o situación estresante.

Lazarus y Folkman, citados por Sandin ${ }^{17}$, se destacan dentro de la línea de investigación que se basa en sucesos menores, donde definieron estrés como un conjunto de relaciones particulares entre la persona y la situación, siendo está valorada por la persona como algo que agrava o excede sus propios recursos y que pone en peligro su bienestar. Esto quiere decir, que el estrés es producto de situaciones donde la persona considera que sus capacidades son sobrepasadas y con ello ve amenazada su tranquilidad.

Richard Lazarus ${ }^{18}$, lo define como el resultado de la relación entre individuo y el entorno, evaluado por aquel como amenazante, que desbordan sus recursos y pone en peligro su bienestar. 
Por otro lado, la Organización Mundial de la Salud (OMS) determina que estrés es un conjunto de reacciones fisiológicas que preparan el organismo para la acción. La organización Internacional del Trabajo ${ }^{19}$, se refiere al estrés laboral bajo los siguientes términos; esta enfermedad es un peligro para las economías de los países industrializados y en vías de desarrollo. Resiente la productividad, al afectar la salud física y mental de los trabajadores.

\section{Karasek, 1979. Modelo de interacción entre demandas y control $^{20}$}

El modelo se basa en las características psicosociales del trabajo, las demandas psicológicas que éste plantea y una cierta combinación de control de las tareas y uso de las capacidades.

- Demandas psicológicas: Son las exigencias que el trabajo implica para la persona. Se refiere a cantidad o volumen de trabajo, presión de tiempo nivel de tensión en cualquier tipo de tipo de tarea.

- Control: Es el factor central del modelo, ya que el este es un recurso para contener las exigencias del trabajo. Por lo tanto, el estrés no depende del nivel de exigencias, sino de poseer los recursos para controlar estas demandas.

El control tiene directa relación a la forma de trabajar y se compone de dos aspectos. El primero es la autonomía, que se refiere a la capacidad de tomar decisiones de manera independiente y dominar sus propias actividades. El segundo aspecto es el desarrollo de habilidades, que guarda relación con los aprendizajes y creatividad desarrollada, las que permiten contener o decidir en la ejecución de las tareas de un puesto de trabajo.

El modelo plantea que el equilibrio entre demanda y control permite responder a situaciones de estrés, afectando de manera positiva la motivación y el desarrollo personal del trabajador.

$\operatorname{Karasek}^{20}$, plantea que el estrés es el resultado de la interacción entre las demandas psicológicas elevadas y la baja libertad de toma de decisiones, es decir, el bajo control. La demanda elevada puede venir por el ritmo y la rapidez del trabajo, por la cantidad de trabajo, por órdenes contradictorias, por demandas conflictivas, por la necesidad de concentración, por la cantidad de interrupciones y por la dependencia del ritmo de los demás. Y el control hace referencia al conjunto de recursos que el trabajador tiene para hacer frente a las demandas; viene determinando tanto su nivel de formación y habilidades como su grado de autonomía y de participación en la toma de decisiones sobre aquellos aspectos que afectan a su trabajo.

De la figura $\mathrm{N}^{\circ} 1$ se puede inferir, que las tareas de alta exigencia son las que ocasionan un mayor nivel de estrés y son dañinos para la salud del trabajador, debido al bajo nivel de control se dificulta cumplir con elevadas demandas. De manera contra puesta, los trabajos de bajo nivel de tensión, proporcionan bajos niveles de estrés.

\section{Karasek y Theorell (1990). Modelo de interacción entre demandas, control y apoyo social.}

Jhonson y Hall ${ }^{21}$ y Karasek y Theorell ${ }^{22}$ amplían el modelo de interacción demandas-control, introduciendo la dimensión de apoyo social como moduladora, de tal forma que un nivel alto de apoyo social en el trabajo disminuye el efecto del estrés, mientras un nivel bajo lo aumenta. El tercer factor modificador es la cantidad y calidad de apoyo social que pueden dar los superiores y compañeros de trabajo. Cuando existe, y es adecuado puede amortiguar parte del potencial del estresor generado por la combinación de las altas
Figura 1.

Modelo interacción demanda y control.

\section{EXIGENCIA PSICOLÓGICA}

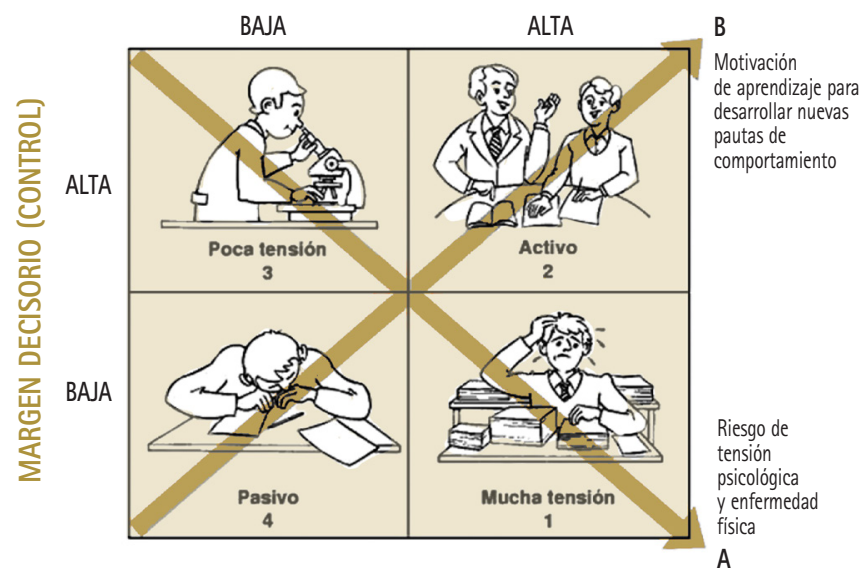

Fuente: Karasek, 1979

demandas o exigencias y el bajo control. Desde este modelo la prevención del estrés laboral se realizaría optimizando las exigencias laborales, aumentando el control del trabajador sobre sus condiciones laborales e incrementando el apoyo social de jefes, subordinados y compañeros. El apoyo social ha sido utilizado en muy diversos sentidos, como red social, como contactos sociales significativos, como posibilidad de tener personas confidentes a las que se puede expresar sentimientos íntimos y como compañía humana. Y tiene una función positiva generalizada sobre la salud y una función amortiguadora sobre el estrés.

Figura 2.

Relación entre demanda psicológica, control y apoyo social.

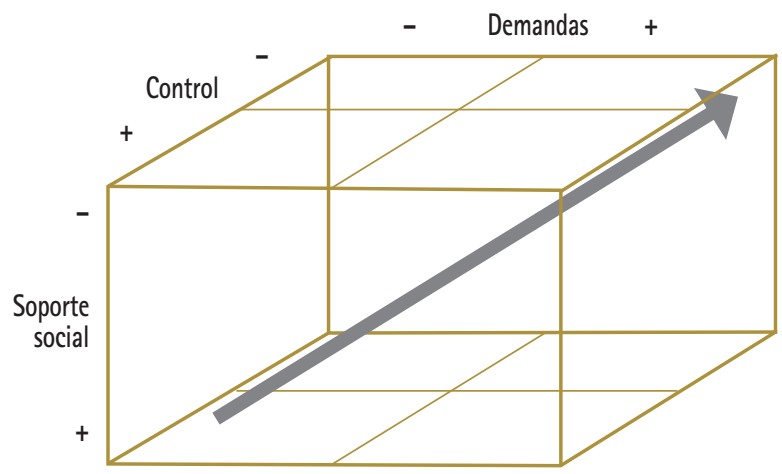

Fuente: Mansilla F. Manual de riesgos psicosociales en el trabajo: Teoría y práctica. ${ }^{23}$

\section{Relación entre las variables de estudio}

Varios estudios indican que la satisfacción laboral se asocia negativamente con diferentes lugares de trabajo donde se percibe un clima de estrés. ${ }^{24-26}$

Beehr y Newman ${ }^{27}$ llegan a la conclusión de que existe una amplia evidencia para sugerir que los factores de estrés en el entorno laboral pueden conducir a disfunción psicológica, que posteriormente dan lugar a efectos adversos en los empleados como la reducción de la satisfacción laboral.

El estrés tiene un efecto negativo en la salud y la satisfacción laboral de sus empleados, y a su vez perjudica la organización en la que 
trabajan. Esto sucede ya que los niveles de productividad y el rendimiento han demostrado estar estrechamente relacionados con el bienestar de los empleados. ${ }^{28}$

Una característica común en los trabajos que presentan niveles de estrés es que éstos generan cambios físicos, psicológicos o de comportamientos negativos en el individuo. ${ }^{29}$

En cuanto a la seguridad, se ha demostrado en estudios que la inversión en la seguridad del trabajo, es el factor más importante para el nivel de trabajo de estrés y el bienestar entre el personal. ${ }^{30}$ Es decir una percepción de riesgo, lo cual lleva a un clima de seguridad negativo, aumentaría la experiencia de estrés y reduce el bienestar psicológico. ${ }^{31}$

Otras teorías sugieren que el estrés y la insatisfacción resultante de la inseguridad laboral conducirán a una bajada del conocimiento y la motivación de seguridad. ${ }^{32,33}$ Según Hofmann y Stetzer ${ }^{34}$, una de las consecuencias de esto puede ser que los empleados tienden a centrar su atención en el rendimiento en lugar de la seguridad en tiempos de estrés, como la posibilidad de poder perder sus puestos de trabajo.

\section{METODOLOGIA}

Para lograr el objetivo se desarrolla un estudio empírico transversal, descriptivo correlacional.

\section{Descripción de la Muestra}

El universo del estudio estuvo conformado por un número de 184 trabajadores de una Institución de Beneficencia de la Provincia de Concepción, los que se dividen en los siguientes grupos de trabajo: calle, infanto adolescente, adulto mayor, educación inicial y el área administrativa. Dentro de éstos grupos de mencionados encontramos un total de 9 profesiones entre las que cuentan Psicólogo, Asistente Social, Auxiliar Paramédico, Educadora de Párvulos, Técnico Educación Parvularia, Asistente de Adulto Mayor, Asistente Hospedería, Técnico en Administración y Manipuladora de Alimentos.

\section{Aplicación del Instrumento}

Con el propósito de evaluar la relación existente entre el nivel de estrés laboral y satisfacción laboral percibido por los trabajadores de la Institución de Beneficencia, se ejecuta la evaluación mediante la aplicación de un instrumento que describe a continuación:
Cuestionario compuesto por 71 preguntas cerradas. Este consta de 3 secciones orientadas a obtener la información necesaria para el estudio:

- Primera sección: identifica antecedentes personales de los encuestados para diferenciarlos (Genero, edad, antigüedad laboral, tipo de contrato, línea de trabajo, profesión).

- La segunda sección orientada a estrés laboral basado en el instrumento de Karasek de 1967, comprende desde la pregunta 1 a la 27. Las preguntas 1-24 son de tipo escala de Likert del 1 al 4 que oscilan desde totalmente en desacuerdo, hasta totalmente de acuerdo. Las preguntas 24 a la 27 incluyen el concepto inseguridad laboral, se responden según estabilidad del empleo, frecuencia y probabilidad de ocurrencia de despidos en su cargo. El instrumento es denominado Job Content Questionnaire (JCQ) o Cuestionario de Contenido del Trabajo, el cual fue diseñado para medir algunos aspectos psicológicos y sociales de las tareas del trabajo, los cuales, de acuerdo con las principales conceptualizaciones teóricas del Modelo Demanda-Control generan tensión laboral. Los derechos de uso del JCQ son reservados, no obstante, el proyecto multicéntrico que coordina el profesor Juárez-García tiene autorización de su uso directo. La traducción original al español fue realizada por Cedillo en el año 2005 y autorizada por el JCQ Center.

- La tercera sección se encuentra orientada a evaluar la percepción de satisfacción laboral de los funcionarios, cuyo cuestionario está desarrollado por Chiang et al en 2008 y 2011. Las preguntas son formuladas para que el encuestado seleccione la alternativa que más le represente del cuestionario conformado por 44 preguntas en escala Likert, con categorización ordinal con puntuaciones de 1 a 5, donde 1 representa "nada satisfecho" y 5 "muy satisfecho".

\section{ANÁLISIS DE LOS DATOS}

\section{Análisis de Fiabilidad}

Como método de fiabilidad se utilizará el alfa de Crombach, este coeficiente expresa cuánto hay de relación a las respuestas, es decir, es un indicador global entre las respuestas.

En la Tabla $n^{\circ} 3$ se observa, la puntuación Alfa para las escalas correspondientes Satisfacción Laboral, se encuentran entre los valores 0.656 y 0.923 . El coeficiente de fiabilidad que presenten es aceptable, por lo tanto, en general el instrumento puede ser aplicado y la información obtenida se puede analizar.

Tabla 3.

Fiabilidad de las escalas de satisfacción laboral - Alfa de Cronbach.

\begin{tabular}{|c|c|c|c|c|c|c|c|c|}
\hline & \multirow[b]{2}{*}{ Factores de Satisfacción } & \multicolumn{2}{|c|}{$\begin{array}{c}\text { Chiang, Salazar, } \\
\text { Huerta y Nuñez (2008) }\end{array}$} & \multicolumn{2}{|c|}{$\begin{array}{c}\text { Chiang, Salazar, } \\
\text { Martín y Nuñez (2011) }\end{array}$} & \multicolumn{3}{|c|}{$\begin{array}{l}\text { Datos actual Investigación } \\
\text { Fundación de beneficencia chilena }\end{array}$} \\
\hline & & $\begin{array}{c}N^{\circ} \\
\text { de Item }\end{array}$ & $\begin{array}{l}\text { Institución } \\
\text { Pública Chilena }\end{array}$ & $\begin{array}{c}N^{\circ} \\
\text { de Item }\end{array}$ & $\begin{array}{l}\text { pital Público } \\
\text { Chileno }\end{array}$ & $\begin{array}{c}N^{\circ} \\
\text { de Item }\end{array}$ & $\begin{array}{l}\text { Alfa de } \\
\text { Cronbach }\end{array}$ & $\begin{array}{c}\text { Alfa de Cronbach } \\
\text { (Si se elimina un ítem) }\end{array}$ \\
\hline I & Satisfacción por el trabajo en general & 10 & 0,910 & 11 & 0,914 & 8 & 0,921 & NO \\
\hline II & Satisfacción con el ambiente físico & 7 & 0,897 & 7 & 0,895 & 7 & 0,872 & NO \\
\hline III & $\begin{array}{l}\text { Satisfacción con la forma } \\
\text { en cómo realiza el trabajo }\end{array}$ & 6 & 0,868 & 6 & 0,877 & 6 & 0,656 & $\begin{array}{c}\text { Si se elimina el ítem } 6 \\
\text { sube a } 0,889, \\
\text { se decide eliminar. }\end{array}$ \\
\hline IV & $\begin{array}{l}\text { Satisfacción con la oportunidad } \\
\text { de desarrollo }\end{array}$ & 7 & 0,834 & 8 & 0,887 & 6 & 0,847 & NO \\
\hline V & Satisfacción con la relación con jefe & 4 & 0,904 & 4 & 0,924 & 5 & 0,894 & NO \\
\hline VI & Satisfacción con la remuneración & 3 & 0,813 & 3 & 0,876 & 3 & 0,755 & NO \\
\hline VII & Satisfacción con la autonomía & 1 & * & 1 & * & 6 & 0,923 & NO \\
\hline VIII & Satisfacción con el reconocimiento & 1 & $*$ & 1 & * & 1 & - & No se calcula fiabilidad \\
\hline IX & Satisfacción por la sección o área & - & - & - & - & 1 & - & No se calcula fiabilidad \\
\hline \multirow[t]{2}{*}{$x$} & Satisfacción por la empresa & - & - & - & - & 1 & - & No se calcula fiabilidad \\
\hline & Cuestionario Completo & 39 & - & 41 & - & 44 & - & - \\
\hline
\end{tabular}


Tabla 4.

Fiabilidad de los factores de stress.

\begin{tabular}{|c|c|c|c|c|}
\hline & Escalas & $\begin{array}{l}\text { Numero } \\
\text { de ítems }\end{array}$ & $\begin{array}{l}\text { Alpha de } \\
\text { Crombach's }\end{array}$ & $\begin{array}{l}\text { Aumenta el Alpha } \\
\text { de Crombach's si se } \\
\text { elimina un elemento }\end{array}$ \\
\hline I & Utilización de habilidades & 6 & 0,682 & İtem 3 sube a 0,640 \\
\hline \multirow[t]{2}{*}{ II } & Autoridad de decisión & 3 & 0,722 & NO \\
\hline & Control laboral & 9 & - & $\begin{array}{l}\text { No se calcula } \\
\text { fiabilidad, porque es la } \\
\text { suma de escala I y II. }\end{array}$ \\
\hline III & Demandas psicológicas & 5 & 0,413 & $\begin{array}{l}\text { Ítems } 14 \text { sube a } 0,635 \text {, } \\
\text { luego se decide } \\
\text { eliminar los items. }\end{array}$ \\
\hline IV & Apoyo social jefe & 4 & 0,927 & NO \\
\hline \multirow[t]{2}{*}{ V } & Apoyo social compañeros & 4 & 0,916 & NO \\
\hline & Apoyo social laboral global & 8 & - & $\begin{array}{l}\text { No se calcula } \\
\text { fiabilidad, porque es la } \\
\text { suma de escala IV y V. }\end{array}$ \\
\hline VI & Inseguridad en el empleo & 3 & 0,898 & NO \\
\hline
\end{tabular}

En la tabla $n^{\circ} 4$, para todas las escalas de estrés los valores varían desde 0.635 y 0.927 , el coeficiente de fiabilidad que representen es aceptable, en general el instrumento puede ser aplicado y los resultados analizados.

En esta tabla $\mathrm{N}^{\circ} 5$ se determina que el promedio general de Satisfacción laboral en las líneas de trabajo evaluadas es de 3,8 puntos, lo que denota según la escala Likert del instrumento un nivel de mediano de satisfacción laboral, considerando la escala de 1 a 5 donde $1=$ nada satisfecho y 5 = muy satisfecho. Se debe mencionar que de manera individual el grupo con mayor grado de satisfacción es el área administrativa con un promedio 4,1, donde su mayor puntuación es en el constructo de satisfacción por el ambiente físico $(4,44)$ y donde su menor puntaje se encuentra en el constructo satisfacción con la remuneración $(3,93)$. En contraste a lo anterior, se observa que el grupo de trabajo con menor grado de satisfacción es adulto mayor con un promedio de 3,7 puntos, donde su mayor puntuación se encuentra en el constructo satisfacción por la empresa $(3,93)$ y donde su menor puntaje se encuentra en el constructo satisfacción con el reconocimiento $(3,58)$.

En la tabla $n^{\circ} 6$, de acuerdo a la dimensión de Utilización de Habilidades, se observa que de los grupos de trabajo estudiados, todos se encuentran con puntuación homogénea con tendencia alta, en relación a su rango.

En el mismo sentido, la dimensión de Autoridad de Decisión, se observa una tendencia homogénea media en relación a su rango. En cuanto a la dimensión de Control Laboral se observa que de los grupos de trabajo estudiados, todos se encuentran con puntuación homogénea con tendencia a ser alta, en relación a su rango. Para el caso de la dimensión de Apoyo Social Jefe se observa una tendencia homogénea media en relación a su rango.

Asimismo, para la dimensión Apoyo Social Compañeros, se observa que de los grupos de trabajo estudiados, todos se encuentran con puntuación homogénea con tendencia a ser alta, en relación a su rango.

También para el caso de la dimensión Apoyo Social Laboral se observa que de los grupos de trabajo estudiados, todos se encuentran con puntuación homogénea con tendencia a ser alta, en relación a su rango.

Finalmente, en la dimensión Inseguridad en el Empleo se observa que de los grupos de trabajo estudiados, todos se encuentran con puntuación homogénea con tendencia alta, en relación a su rango.
Tabla 5.

Análisis de promedios y desviación Estándar Satisfacción según grupos de trabajo.

\begin{tabular}{|c|c|c|c|c|c|c|}
\hline Escalas / L T & & $\begin{array}{l}\text { Línea } \\
\text { Calle }\end{array}$ & $\begin{array}{c}\text { Infanto } \\
\text { Adolescente }\end{array}$ & $\begin{array}{l}\text { Adulto } \\
\text { Mayor }\end{array}$ & $\begin{array}{l}\text { Educación } \\
\text { Inicial }\end{array}$ & Administración \\
\hline $\begin{array}{l}\text { Satisfacción por el } \\
\text { trabajo en general }\end{array}$ & $\begin{array}{l}p \\
d\end{array}$ & $\begin{array}{l}4,08 \\
0,55\end{array}$ & $\begin{array}{l}4,06 \\
0,66\end{array}$ & $\begin{array}{l}3,67 \\
0,93\end{array}$ & $\begin{array}{l}3,96 \\
0,62\end{array}$ & $\begin{array}{l}4,21 \\
0,28\end{array}$ \\
\hline $\begin{array}{l}\text { Satisfacción con el } \\
\text { ambiente físico }\end{array}$ & $\begin{array}{l}p \\
d\end{array}$ & $\begin{array}{l}3,77 \\
0,56\end{array}$ & $\begin{array}{l}4,20 \\
0,50\end{array}$ & $\begin{array}{l}3,78 \\
0,85\end{array}$ & $\begin{array}{l}3,80 \\
0,64\end{array}$ & $\begin{array}{l}4,44 \\
0,42\end{array}$ \\
\hline $\begin{array}{l}\text { Satisfacción con la } \\
\text { forma en cómo } \\
\text { realiza el trabajo }\end{array}$ & $\begin{array}{l}p \\
d\end{array}$ & $\begin{array}{l}3,78 \\
0,68\end{array}$ & $\begin{array}{l}3,93 \\
0,61\end{array}$ & $\begin{array}{l}3,81 \\
0,91\end{array}$ & $\begin{array}{l}4,06 \\
0,60\end{array}$ & $\begin{array}{l}4,23 \\
0,32\end{array}$ \\
\hline $\begin{array}{l}\text { Satisfacción con la } \\
\text { oportunidad de } \\
\text { desarrollo }\end{array}$ & $\begin{array}{l}p \\
d\end{array}$ & $\begin{array}{l}3,82 \\
0,55\end{array}$ & $\begin{array}{l}3,85 \\
0,72\end{array}$ & $\begin{array}{l}3,68 \\
0,87\end{array}$ & $\begin{array}{l}3,76 \\
0,61\end{array}$ & $\begin{array}{l}4,07 \\
0,25\end{array}$ \\
\hline $\begin{array}{l}\text { Satisfacción con la } \\
\text { relación con jefe }\end{array}$ & $\begin{array}{l}p \\
d\end{array}$ & $\begin{array}{l}4,09 \\
0,53\end{array}$ & $\begin{array}{l}3,99 \\
0,71\end{array}$ & $\begin{array}{l}3,86 \\
0,88\end{array}$ & $\begin{array}{l}3,99 \\
0,60\end{array}$ & $\begin{array}{l}4,18 \\
0,45\end{array}$ \\
\hline $\begin{array}{l}\text { Satisfacción con } \\
\text { la remuneración }\end{array}$ & $\begin{array}{l}p \\
d\end{array}$ & $\begin{array}{l}3,40 \\
0,77\end{array}$ & $\begin{array}{l}3,69 \\
0,77\end{array}$ & $\begin{array}{l}3,60 \\
0,88\end{array}$ & $\begin{array}{l}3,51 \\
0,72\end{array}$ & $\begin{array}{l}3,93 \\
0,44\end{array}$ \\
\hline $\begin{array}{l}\text { Satisfacción con } \\
\text { la autonomía }\end{array}$ & p & $\begin{array}{l}3,86 \\
0,46\end{array}$ & $\begin{array}{l}4,12 \\
0,41\end{array}$ & $\begin{array}{l}3,77 \\
0,98\end{array}$ & $\begin{array}{l}3,89 \\
0,65\end{array}$ & $\begin{array}{l}4,10 \\
0,30\end{array}$ \\
\hline $\begin{array}{l}\text { Satisfacción con } \\
\text { el reconocimiento }\end{array}$ & $\begin{array}{l}p \\
d\end{array}$ & $\begin{array}{l}3,63 \\
0,83\end{array}$ & $\begin{array}{l}3,90 \\
1,05\end{array}$ & $\begin{array}{l}3,58 \\
1,26\end{array}$ & $\begin{array}{l}3,78 \\
0,90\end{array}$ & $\begin{array}{l}4,40 \\
0,52\end{array}$ \\
\hline $\begin{array}{l}\text { Satisfacción por la } \\
\text { sección o área }\end{array}$ & $\begin{array}{l}\mathrm{p} \\
\mathrm{d}\end{array}$ & $\begin{array}{l}4,05 \\
0,71\end{array}$ & $\begin{array}{l}4,21 \\
0,68\end{array}$ & $\begin{array}{l}3,83 \\
1,04\end{array}$ & $\begin{array}{l}3,79 \\
0,75\end{array}$ & $\begin{array}{l}4,20 \\
0,63\end{array}$ \\
\hline $\begin{array}{l}\text { Satisfacción por } \\
\text { la empresa }\end{array}$ & $\begin{array}{l}p \\
d\end{array}$ & $\begin{array}{l}4,26 \\
0,81\end{array}$ & $\begin{array}{l}4,14 \\
0,64\end{array}$ & $\begin{array}{l}3,93 \\
1,01\end{array}$ & $\begin{array}{l}3,99 \\
0,71\end{array}$ & $\begin{array}{l}4,10 \\
0,32\end{array}$ \\
\hline
\end{tabular}

Tabla 6.

Análisis de promedios y desviación Estándar Estrés según Grupos de Trabajo.

$\begin{array}{lccccccc}\text { Escalas / L T } & & \begin{array}{c}\text { Línea } \\ \text { Calle }\end{array} & \begin{array}{c}\text { Infanto } \\ \text { Adolescente }\end{array} & \begin{array}{c}\text { Adulto } \\ \text { Mayor }\end{array} & \begin{array}{c}\text { Educación } \\ \text { Inicial }\end{array} & \begin{array}{c}\text { Adminis- } \\ \text { tración }\end{array} & \text { Rango } \\ \text { Utilización de } & \text { p } & 37,58 & 36,76 & 35,76 & 37,94 & 35,60 & 12-48 \\ \text { habilidades } & \text { d } & 4,09 & 2,69 & 6,19 & 4,09 & 3,63 & \\ \text { Autoridad de } & \text { p } & 36,21 & 35,45 & 35,80 & 37,85 & 33,60 & 12-48 \\ \text { decisión } & \text { d } & 6,32 & 4,24 & 7,59 & 6,54 & 5,40 & \\ \text { Demandas } & \text { p } & 28,16 & 26,21 & 25,03 & 26,61 & 28,70 & 12-48 \\ \text { psicológicas } & \text { d } & 5,52 & 4,01 & 5,56 & 4,39 & 3,53 & \\ \text { Control Laboral } & \text { p } & 73,79 & 72,21 & 71,56 & 75,79 & 69,20 & 24-96 \\ & \text { d } & 9,09 & 5,27 & 12,70 & 9,68 & 7,84 & \\ \text { Apoyo social Jefe } & \text { p } & 13,58 & 12,69 & 12,32 & 14,00 & 12,70 & 4-16 \\ & \text { d } & 2,50 & 2,79 & 3,22 & 2,43 & 1,57 & \\ \text { Apoyo social } & \text { p } & 13,32 & 13,31 & 11,63 & 13,60 & 13,00 & 4-16 \\ \text { Compañeros } & \text { d } & 2,79 & 2,12 & 3,56 & 2,77 & 1,63 & \\ \text { Apoyo social } & \text { p } & 26,89 & 26,00 & 23,95 & 27,60 & 25,70 & 4-32 \\ \text { Laboral Global } & \text { d } & 4,83 & 4,42 & 6,33 & 4,91 & 2,83 & \\ \text { Inseguridad en } & \text { p } & 6,32 & 7,59 & 6,32 & 6,18 & 6,00 & 1-8 \\ \text { el empleo } & \text { d } & 0,75 & 1,82 & 1,59 & 1,17 & 0,82 & \end{array}$

Figura 7.

Modelo de Karasek aplicado a los Grupos de Trabajo de la Fundación de Beneficencia.

Relación Demanda-Control de Karasek

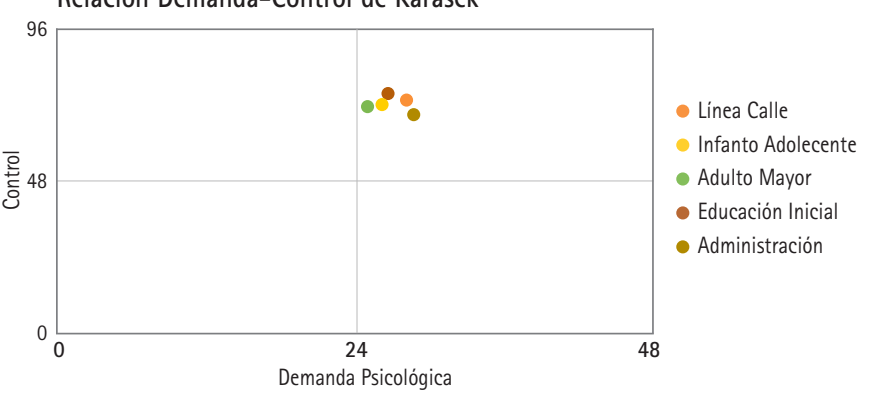

Fuente: Elaboración propia. 


\section{Figura 8.}

Modelo demanda-control-apoyo social laboral global aplicado a los Grupos de Trabajo de la Fundación de Beneficencia.

Relación Demanda-Control-Apoyo Social Global

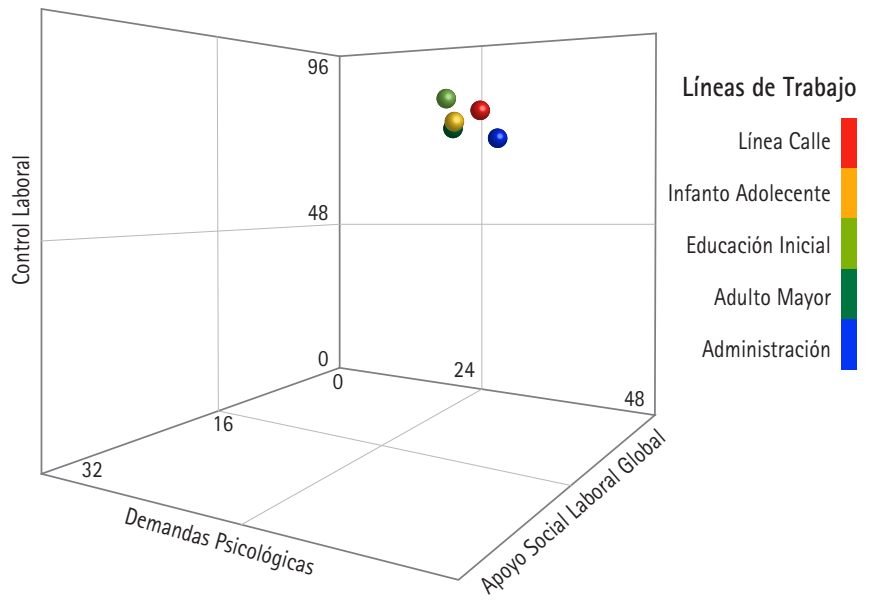

Fuente: Elaboración propia.

Es posible concluir que la muestra tuvo un comportamiento en general homogéneo, sin observarse Grupos de Trabajo que hayan marcado alguna tendencia significativa.

En la figura $N^{\circ} 7$ se observa que los funcionarios que trabajan en el grupo de educación inicial perciben mayores demandas psicológicas y a su vez mayor capacidad de control laboral respecto de quienes se desempeñan en el grupo de trabajo de Administración en la Fundación de Beneficencia. Sin embargo, todos se ubican en el cuadrante activo del modelo.

En la figura $\mathrm{N}^{\circ} 8$ se observa el modelo demanda- control asociado a una tercera dimensión "apoyo social global" incorporada por Jeffrey V. Johnson en 1986, la cual refleja el apoyo social de los compañeros y jefes percibido por los funcionarios.
Al incluir esta dimensión en el análisis, los datos presentan una disminución en las demandas psicológicas percibidas por los funcionarios de los distintos grupos de trabajo de la Fundación de Beneficencia, reubicando los resultados dentro del cuadrante "Poca Tensión" del modelo. Se observa además, que en el grupo de trabajo de educación inicial mantiene la percepción de mayor control laboral en relación a sus pares de otros grupos de trabajo.

\section{Análisis de Correlaciones entre Satisfacción y Estrés Laboral}

Con el objetivo de analizar la relación entre las dimensiones de la escala de estrés laboral y las dimensiones de la escala de satisfacción laboral, se calculó el coeficiente de correlación Rho de Spearman entre tales dimensiones. Es pertinente recordar que en la escala de estrés, la variable control laboral es la suma de las dimensiones utilización de habilidades y autoridad de decisión, mientras que la variable apoyo social laboral global es la suma de las dimensiones apoyo social del jefe y apoyo social de los compañeros.

La Tabla $\mathrm{N}^{\circ} 9$ refleja los resultados obtenidos en la muestra total, se observa que la dimensión demandas psicológicas se relaciona estadísticamente significativa de manera negativa con la satisfacción con la forma en la que realiza el trabajo y con la satisfacción con la autonomía. Lo anterior señala que a mayor percepción de satisfacción, con la forma de realizar el trabajo y con la autonomía, menor es la percepción de demanda psicológica.

En cuanto a la dimensión de Control Laboral se relaciona estadísticamente significativa positiva con la Satisfacción por el Trabajo en general, Satisfacción con el ambiente físico, Satisfacción en la forma como realiza el trabajo, Satisfacción con la oportunidad de desarrollo, Satisfacción con la remuneración, Satisfacción con el reconocimiento, Satisfacción por la sección o área, Satisfacción por la empresa, Satisfacción con la relación con jefe y Satisfacción con la autonomía. Dando como resultado, que a mayor percepción de las variables de Satisfacción y mayor Control sobre el trabajo, se logra reducir el grado de estrés.

Tabla 9.

Correlación satisfacción vs estrés. Muestra Total.

\begin{tabular}{|c|c|c|c|c|c|c|c|c|}
\hline & & $\begin{array}{l}\text { Utilización de } \\
\text { habilidades }\end{array}$ & $\begin{array}{l}\text { Autoridad } \\
\text { de decisión }\end{array}$ & $\begin{array}{l}\text { Demandas } \\
\text { psicológicas }\end{array}$ & $\begin{array}{l}\text { Control } \\
\text { laboral }\end{array}$ & $\begin{array}{c}\text { Apoyo } \\
\text { social jefe }\end{array}$ & $\begin{array}{l}\text { Apoyo social } \\
\text { compañeros }\end{array}$ & $\begin{array}{l}\text { Apoyo social } \\
\text { laboral global }\end{array}$ \\
\hline $\begin{array}{l}\text { Satisfacción por } \\
\text { el trabajo en general }\end{array}$ & $\begin{array}{l}\text { Correlación } \\
\text { Significancia }\end{array}$ & $\begin{array}{c}0,330^{* *} \\
0,000\end{array}$ & $\begin{array}{c}0,325^{* *} \\
0,000\end{array}$ & $\begin{array}{c}-0,048 \\
0,520\end{array}$ & $\begin{array}{c}0,375^{* *} \\
0,000\end{array}$ & $\begin{array}{c}0,501^{* *} \\
0,000\end{array}$ & $\begin{array}{c}0,487^{* *} \\
0,000\end{array}$ & $\begin{array}{c}0,556^{* *} \\
0,000\end{array}$ \\
\hline Satisfacción con el ambiente físico & $\begin{array}{l}\text { Correlación } \\
\text { Significancia }\end{array}$ & $\begin{array}{l}0,157^{*} \\
0,033\end{array}$ & $\begin{array}{c}0,205^{* *} \\
0,005\end{array}$ & $\begin{array}{c}-0,010 \\
0,890\end{array}$ & $\begin{array}{c}0,213^{* *} \\
0,004\end{array}$ & $\begin{array}{c}0,254^{* *} \\
, 001\end{array}$ & $\begin{array}{c}0,288^{* *} \\
0,000\end{array}$ & $\begin{array}{c}0,309^{* *} \\
0,000\end{array}$ \\
\hline $\begin{array}{l}\text { Satisfacción con la forma } \\
\text { en cómo realiza el trabajo }\end{array}$ & $\begin{array}{l}\text { Correlación } \\
\text { Significancia }\end{array}$ & $\begin{array}{c}0,252^{* *} \\
0,001\end{array}$ & $\begin{array}{c}0,298^{* *} \\
0,000\end{array}$ & $\begin{array}{c}-0,147^{*} \\
0,046\end{array}$ & $\begin{array}{c}0,320^{* *} \\
0,000\end{array}$ & $\begin{array}{c}0,427^{* *} \\
0,000\end{array}$ & $\begin{array}{c}0,342^{* *} \\
0,000\end{array}$ & $\begin{array}{c}0,417^{* *} \\
0,000\end{array}$ \\
\hline $\begin{array}{l}\text { Satisfacción con la oportunidad } \\
\text { de desarrollo }\end{array}$ & $\begin{array}{l}\text { Correlación } \\
\text { Significancia }\end{array}$ & $\begin{array}{c}0,296^{* *} \\
0,000\end{array}$ & $\begin{array}{c}0,247^{* *} \\
0,001\end{array}$ & $\begin{array}{c}-0,050 \\
0,499\end{array}$ & $\begin{array}{c}0,301^{* *} \\
0,000\end{array}$ & $\begin{array}{c}0,456^{* *} \\
0,000\end{array}$ & $\begin{array}{c}0,404^{* *} \\
0,000\end{array}$ & $\begin{array}{c}0,483^{* *} \\
0,000\end{array}$ \\
\hline Satisfacción con la relación con jefe & $\begin{array}{l}\text { Correlación } \\
\text { Significancia }\end{array}$ & $\begin{array}{c}0,276^{* *} \\
0,000\end{array}$ & $\begin{array}{c}0,360^{* *} \\
0,000\end{array}$ & $\begin{array}{c}-0,109 \\
0,142\end{array}$ & $\begin{array}{c}0,372^{* *} \\
0,000\end{array}$ & $\begin{array}{c}0,515^{* *} \\
0,000\end{array}$ & $\begin{array}{c}0,413^{* *} \\
0,000\end{array}$ & $\begin{array}{c}0,524^{* *} \\
0,000\end{array}$ \\
\hline Satisfacción con la remuneración & $\begin{array}{l}\text { Correlación } \\
\text { Significancia }\end{array}$ & $\begin{array}{l}0,113 \\
0,126\end{array}$ & $\begin{array}{c}0,191^{* *} \\
0,009\end{array}$ & $\begin{array}{l}-0,125 \\
0,091\end{array}$ & $\begin{array}{c}0,188^{*} \\
0,011\end{array}$ & $\begin{array}{c}0,219^{* *} \\
0,003\end{array}$ & $\begin{array}{c}0,197^{* *} \\
0,007\end{array}$ & $\begin{array}{c}0,223^{* *} \\
0,002\end{array}$ \\
\hline Satisfacción con la autonomía & $\begin{array}{l}\text { Correlación } \\
\text { Significancia }\end{array}$ & $\begin{array}{c}0,328^{* *} \\
0,000\end{array}$ & $\begin{array}{c}0,353^{* *} \\
0,000\end{array}$ & $\begin{array}{c}-0,166^{*} \\
0,024\end{array}$ & $\begin{array}{c}0,396^{* *} \\
0,000\end{array}$ & $\begin{array}{c}0,474^{* *} \\
0,000\end{array}$ & $\begin{array}{c}0,425^{* *} \\
0,000\end{array}$ & $\begin{array}{c}0,495^{* *} \\
0,000\end{array}$ \\
\hline Satisfacción con el reconocimiento & $\begin{array}{l}\text { Correlación } \\
\text { Significancia }\end{array}$ & $\begin{array}{c}0,273^{* *} \\
0,000\end{array}$ & $\begin{array}{c}0,268^{* *} \\
0,000\end{array}$ & $\begin{array}{c}-0,013 \\
0,861\end{array}$ & $\begin{array}{c}0,316^{* *} \\
0,000\end{array}$ & $\begin{array}{c}0,382^{* *} \\
0,000\end{array}$ & $\begin{array}{c}0,257^{* *} \\
0,000\end{array}$ & $\begin{array}{c}0,351^{* *} \\
0,000\end{array}$ \\
\hline Satisfacción por la sección o área & $\begin{array}{l}\text { Correlación } \\
\text { Significancia }\end{array}$ & $\begin{array}{c}0,315^{* *} \\
0,000\end{array}$ & $\begin{array}{c}0,220^{* *} \\
0,003\end{array}$ & $\begin{array}{c}-0,140 \\
0,059\end{array}$ & $\begin{array}{c}0,292^{* *} \\
0,000\end{array}$ & $\begin{array}{c}0,290^{* *} \\
0,000\end{array}$ & $\begin{array}{c}0,313^{* *} \\
0,000\end{array}$ & $\begin{array}{c}0,338^{* *} \\
0,000\end{array}$ \\
\hline Satisfacción por la empresa & $\begin{array}{l}\text { Correlación } \\
\text { Significancia }\end{array}$ & $\begin{array}{l}0,078 \\
0,295\end{array}$ & $\begin{array}{c}0,201^{* *} \\
0,006\end{array}$ & $\begin{array}{c}-0,120 \\
0,104\end{array}$ & $\begin{array}{c}0,178^{*} \\
0,016\end{array}$ & $\begin{array}{c}0,399^{* *} \\
0,000\end{array}$ & $\begin{array}{c}0,341^{* *} \\
0,000\end{array}$ & $\begin{array}{c}0,422^{* *} \\
0,000\end{array}$ \\
\hline
\end{tabular}

**. Correlation is significant at the 0,01 level (2-tailed). *. Correlation is significant at the 0,05 level (2-tailed). Spearman's rho. 
Por otro lado, la dimensión de Apoyo Social Laboral Global se relaciona estadísticamente significativa positiva con Satisfacción por el trabajo en general, Satisfacción con la Relación con Jefe y Satisfacción con autonomía. Lo anterior, es congruente con lo analizado hasta ahora; ya que el grado de Apoyo Social Laboral Global condiciona positivamente con el grado de satisfacción percibido por el trabajador.

La Tabla $\mathrm{N}^{\circ} 10$ refleja los resultados obtenidos en el tipo de contrato indefinido, se observa que la dimensión Demandas Psicológicas no tiene relación estadísticamente significativa con ninguna de las dimensiones de Satisfacción laboral.
En cuanto a la dimensión Control Laboral, tiene relación estadísticamente significativa positiva con las dimensiones de Satisfacción por el trabajo en general, Satisfacción con la relación con jefe y Satisfacción con la autonomía. Dando como resultado, que a mayor percepción de las variables de Satisfacción y mayor Control sobre el trabajo, se logra reducir el grado de estrés. Siendo lo anterior similar a lo que se aprecia en la muestra total.

La dimensión de Apoyo Social Laboral Global, se relacionan estadísticamente significativa positiva con las dimensiones de Satisfacción por el trabajo en general, Satisfacción con la oportunidad de desarrollo, Satisfacción con la relación con jefe y

Tabla 10.

Correlación Satisfacción vs Estrés por contrato indefinido.

\begin{tabular}{|c|c|c|c|c|c|c|c|c|}
\hline & & $\begin{array}{l}\text { Utilización } \\
\text { de habilidades }\end{array}$ & $\begin{array}{l}\text { Autoridad } \\
\text { de decisión }\end{array}$ & $\begin{array}{l}\text { Demandas } \\
\text { psicológicas }\end{array}$ & $\begin{array}{l}\text { Control } \\
\text { laboral }\end{array}$ & $\begin{array}{c}\text { Apoyo } \\
\text { social jefe }\end{array}$ & $\begin{array}{l}\text { Apoyo social } \\
\text { compañeros }\end{array}$ & $\begin{array}{l}\text { Apoyo social } \\
\text { laboral global }\end{array}$ \\
\hline Satisfacción por el trabajo en general & $\begin{array}{l}\text { Correlación } \\
\text { Significancia }\end{array}$ & $\begin{array}{c}0,333^{* *} \\
0,000\end{array}$ & $\begin{array}{c}0,321^{* *} \\
0,000\end{array}$ & $\begin{array}{l}0,009 \\
0,907\end{array}$ & $\begin{array}{c}0,364^{* *} \\
0,000\end{array}$ & $\begin{array}{c}0,517^{* *} \\
0,000\end{array}$ & $\begin{array}{c}0,500^{* *} \\
0,000\end{array}$ & $\begin{array}{c}0,568^{* *} \\
0,000\end{array}$ \\
\hline Satisfacción con el ambiente físico & $\begin{array}{l}\text { Correlación } \\
\text { Significancia }\end{array}$ & $\begin{array}{l}0,178^{*} \\
0,023\end{array}$ & $\begin{array}{c}0,206^{* *} \\
0,008\end{array}$ & $\begin{array}{l}0,067 \\
0,395\end{array}$ & $\begin{array}{c}0,220^{* *} \\
0,005\end{array}$ & $\begin{array}{c}0,297^{* *} \\
0,000\end{array}$ & $\begin{array}{c}0,309^{* *} \\
0,000\end{array}$ & $\begin{array}{c}0,341^{* *} \\
0,000\end{array}$ \\
\hline $\begin{array}{l}\text { Satisfacción con la forma en } \\
\text { cómo realiza el trabajo }\end{array}$ & $\begin{array}{l}\text { Correlación } \\
\text { Significancia }\end{array}$ & $\begin{array}{c}0,240^{* *} \\
0,002\end{array}$ & $\begin{array}{c}0,299^{* *} \\
0,000\end{array}$ & $\begin{array}{l}-0,088 \\
0,263\end{array}$ & $\begin{array}{c}0,304^{* *} \\
0,000\end{array}$ & $\begin{array}{c}0,414^{* *} \\
0,000\end{array}$ & $\begin{array}{c}0,318^{* *} \\
0,000\end{array}$ & $\begin{array}{c}0,392^{* *} \\
0,000\end{array}$ \\
\hline $\begin{array}{l}\text { Satisfacción con la } \\
\text { oportunidad de desarrollo }\end{array}$ & $\begin{array}{l}\text { Correlación } \\
\text { Significancia }\end{array}$ & $\begin{array}{c}0,299^{* *} \\
0,000\end{array}$ & $\begin{array}{c}0,235^{* *} \\
0,002\end{array}$ & $\begin{array}{l}0,015 \\
0,847\end{array}$ & $\begin{array}{c}0,284^{* *} \\
0,000\end{array}$ & $\begin{array}{c}0,461^{* *} \\
0,000\end{array}$ & $\begin{array}{c}0,411^{* *} \\
0,000\end{array}$ & $\begin{array}{c}0,486^{* *} \\
0,000\end{array}$ \\
\hline Satisfacción con la relación con jefe & $\begin{array}{l}\text { Correlación } \\
\text { Significancia }\end{array}$ & $\begin{array}{c}0,281^{* *} \\
0,000\end{array}$ & $\begin{array}{c}0,362^{* *} \\
0,000\end{array}$ & $\begin{array}{c}-0,044 \\
0,579\end{array}$ & $\begin{array}{c}0,367^{* *} \\
0,000\end{array}$ & $\begin{array}{c}0,511^{* *} \\
0,000\end{array}$ & $\begin{array}{c}0,412^{* *} \\
0,000\end{array}$ & $\begin{array}{c}0,518^{* *} \\
0,000\end{array}$ \\
\hline Satisfacción con la remuneración & $\begin{array}{l}\text { Correlación } \\
\text { Significancia }\end{array}$ & $\begin{array}{l}0,118 \\
0,132\end{array}$ & $\begin{array}{l}0,191^{*} \\
0,014\end{array}$ & $\begin{array}{c}-0,096 \\
0,221\end{array}$ & $\begin{array}{c}0,183^{*} \\
0,019\end{array}$ & $\begin{array}{c}0,254^{* *} \\
0,001\end{array}$ & $\begin{array}{c}0,195^{*} \\
0,012\end{array}$ & $\begin{array}{c}0,239^{* *} \\
0,002\end{array}$ \\
\hline Satisfacción con la autonomía & $\begin{array}{l}\text { Correlación } \\
\text { Significancia }\end{array}$ & $\begin{array}{c}0,319^{* *} \\
0,000\end{array}$ & $\begin{array}{c}0,354^{* *} \\
0,000\end{array}$ & $\begin{array}{c}-0,124 \\
0,114\end{array}$ & $\begin{array}{c}0,384^{* *} \\
0,000\end{array}$ & $\begin{array}{c}0,479^{* *} \\
0,000\end{array}$ & $\begin{array}{c}0,413^{* *} \\
0,000\end{array}$ & $\begin{array}{c}0,488^{* *} \\
0,000\end{array}$ \\
\hline Satisfacción con el reconocimiento & $\begin{array}{l}\text { Correlación } \\
\text { Significancia }\end{array}$ & $\begin{array}{c}0,245^{* *} \\
0,002\end{array}$ & $\begin{array}{c}0,267^{* *} \\
0,001\end{array}$ & $\begin{array}{l}0,012 \\
0,882\end{array}$ & $\begin{array}{c}0,294^{* *} \\
0,000\end{array}$ & $\begin{array}{c}0,408^{* *} \\
0,000\end{array}$ & $\begin{array}{c}0,261^{* *} \\
0,001\end{array}$ & $\begin{array}{c}0,365^{* *} \\
0,000\end{array}$ \\
\hline Satisfacción por la sección o área & $\begin{array}{l}\text { Correlación } \\
\text { Significancia }\end{array}$ & $\begin{array}{c}0,306^{* *} \\
0,000\end{array}$ & $\begin{array}{c}0,246^{* *} \\
0,002\end{array}$ & $\begin{array}{l}-0,110 \\
0,162\end{array}$ & $\begin{array}{c}0,299^{* *} \\
0,000\end{array}$ & $\begin{array}{c}0,311^{* *} \\
0,000\end{array}$ & $\begin{array}{c}0,313^{* *} \\
0,000\end{array}$ & $\begin{array}{c}0,350^{* *} \\
0,000\end{array}$ \\
\hline Satisfacción por la empresa & $\begin{array}{l}\text { Correlación } \\
\text { Significancia }\end{array}$ & $\begin{array}{c}0,035 \\
0,660\end{array}$ & $\begin{array}{c}0,183^{*} \\
0,019\end{array}$ & $\begin{array}{c}-0,084 \\
0,287\end{array}$ & $\begin{array}{l}0,138 \\
0,078\end{array}$ & $\begin{array}{c}0,370^{* *} \\
0,000\end{array}$ & $\begin{array}{c}0,330^{* *} \\
0,000\end{array}$ & $\begin{array}{c}0,399^{* *} \\
0,000\end{array}$ \\
\hline
\end{tabular}

**. Correlation is significant at the 0,01 level (2-tailed). * *. Correlation is significant at the 0,05 level (2-tailed). Spearman's rho.

Tabla 11.

Correlación Satisfacción vs Estrés por contrato a plazo.

\begin{tabular}{|c|c|c|c|c|c|c|c|c|}
\hline & & $\begin{array}{l}\text { Utilización } \\
\text { de habilidades }\end{array}$ & $\begin{array}{l}\text { Autoridad } \\
\text { de decisión }\end{array}$ & $\begin{array}{l}\text { Demandas } \\
\text { psicológicas }\end{array}$ & $\begin{array}{l}\text { Control } \\
\text { laboral }\end{array}$ & $\begin{array}{c}\text { Apoyo } \\
\text { social jefe }\end{array}$ & $\begin{array}{l}\text { Apoyo social } \\
\text { compañeros }\end{array}$ & $\begin{array}{l}\text { Apoyo social } \\
\text { laboral global }\end{array}$ \\
\hline \multirow{2}{*}{ Satisfacción por el trabajo en general } & Correlación & 00,303 & 0,290 & $-0,473^{*}$ & 0,436 & 0,379 & 0,407 & $0,447^{*}$ \\
\hline & Significancia & 0,193 & 0,215 & 0,035 & 0,055 & 0,099 & 0,075 & 0,048 \\
\hline \multirow{2}{*}{ Satisfacción con el ambiente físico } & Correlación & $-0,031$ & 0,137 & $-0,695^{* *}$ & 0,094 & $-0,126$ & 0,082 & $-0,017$ \\
\hline & Significancia & 0,898 & 0,563 & 0,001 & 0,694 & 0,596 & 0,731 & 0,942 \\
\hline \multirow{2}{*}{$\begin{array}{l}\text { Satisfacción con la forma } \\
\text { en cómo realiza el trabajo }\end{array}$} & Correlación & 0,298 & 0,285 & $-0,613^{* *}$ & 0,437 & $0,509^{*}$ & $0,602^{* *}$ & $0,608^{* *}$ \\
\hline & Significancia & 0,202 & 0,223 & 0,004 & 0,054 & 0,022 & 0,005 & 0,004 \\
\hline \multirow{2}{*}{$\begin{array}{l}\text { Satisfacción con la oportunidad } \\
\text { de desarrollo }\end{array}$} & Correlación & 0,252 & 0,293 & $-0,532^{*}$ & 0,398 & 0,442 & $0,448^{*}$ & $0,495^{*}$ \\
\hline & Significancia & 0,283 & 0,210 & 0,016 & 0,082 & 0,051 & 0,047 & 0,026 \\
\hline \multirow{2}{*}{ Satisfacción con la relación con jefe } & Correlación & 0,258 & 0,359 & $-0,681^{* *}$ & 0,418 & $0,522^{*}$ & $0,484^{*}$ & $0,571^{* *}$ \\
\hline & Significancia & 0,273 & 0,120 & 0,001 & 0,067 & 0,018 & 0,031 & 0,009 \\
\hline \multirow{2}{*}{ Satisfacción con la remuneración } & Correlación & 0,100 & 0,237 & $-0,305$ & 0,259 & 0,021 & 0,246 & 0,127 \\
\hline & Significancia & 0,674 & 0,315 & 0,191 & 0,271 & 0,931 & 0,295 & 0,594 \\
\hline \multirow{2}{*}{ Satisfacción con la autonomía } & Correlación & 0,345 & 0,324 & $-0,525^{*}$ & $0,475^{*}$ & 0,398 & $0,579^{* *}$ & $0,537^{*}$ \\
\hline & Significancia & 0,136 & 0,164 & 0,017 & 0,034 & 0,083 & 0,007 & 0,015 \\
\hline \multirow{2}{*}{ Satisfacción con el reconocimiento } & Correlación & $0,464^{*}$ & 0,244 & $-0,220$ & $0,485^{*}$ & 0,241 & 0,327 & 0,317 \\
\hline & Significancia & 0,040 & 0,300 & 0,350 & 0,030 & 0,307 & 0,160 & 0,174 \\
\hline \multirow{2}{*}{ Satisfacción por la sección o área } & Correlación & 0,413 & 0,035 & $-0,437$ & 0,278 & 0,131 & 0,339 & 0,244 \\
\hline & Significancia & 0,070 & 0,885 & 0,054 & 0,235 & 0,581 & 0,143 & 0,300 \\
\hline \multirow{2}{*}{ Satisfacción por la empresa } & Correlación & 0,381 & 0,345 & $-0,420$ & $0,476^{*}$ & $0,574^{* *}$ & $0,515^{*}$ & $0,607^{* *}$ \\
\hline & Significancia & 0,098 & 0,136 & 0,065 & 0,034 & 0,008 & 0,020 & 0,005 \\
\hline
\end{tabular}

**. Correlation is significant at the 0,01 level (2-tailed). *. Correlation is significant at the 0,05 level (2-tailed). Spearman's rho. 
Satisfacción con la autonomía. Determinando que al estar elevado el Apoyo Social Laboral Global aumente la Satisfacción, permitiendo que el grado de estrés percibido disminuya.

La Tabla $\mathrm{N}^{\circ} 11$ refleja los resultados obtenidos en la muestra de contratos a plazo, se observa la Dimensión de Demandas Psicológicas una relación estadísticamente significativa negativa con las dimensiones de Satisfacción por el trabajo en general, Satisfacción por el ambiente físico, Satisfacción con la forma en como realiza el trabajo, Satisfacción con la oportunidad de desarrollo, Satisfacción con la relación con jefe Satisfacción con la autonomía, Satisfacción por la sección o área y Satisfacción por la empresa. Los resultados indican que a mayor percepción de Satisfacción por el Trabajo en General, menor será la percepción de Demandas Psicológicas.

En cuanto a la dimensión Control Laboral se observa una relación estadísticamente significativa positiva con las dimensiones de Satisfacción con la autonomía, Satisfacción con el reconocimiento y Satisfacción por la empresa. Dando como resultado, que a mayor percepción de las variables de Satisfacción y mayor Control del trabajo, se logra reducir el grado de estrés. Siendo lo anterior similar a lo que se aprecia en la muestra total.

En la dimensión de Apoyo Social Laboral Global, se observa una relación estadísticamente significativa positiva con las dimensiones de Satisfacción por el trabajo en general, Satisfacción con la forma en cómo se realiza el trabajo, Satisfacción con la oportunidad de desarrollo, Satisfacción con la relación con jefe, Satisfacción con la autonomía y Satisfacción por la empresa. Determinando que al estar elevadas el Apoyo Social Laboral Global aumente la Satisfacción, permiten así que el grado de estrés percibido disminuya.

\section{CONCLUSIONES}

Utilizando el Modelo de Demanda-Control, formulado por $\operatorname{Karasek}^{20}$, se puede concluir que a nivel general, los grupos de trabajo calle, infanto adolescente, adulto mayor y educación inicial se posicionan en el cuadrante baja tensión, lo cual indica que los trabajadores experimentan un bajo nivel de estrés y alto control laboral comparado con el grupo administrativo, ésta última posee un nivel de concentración mayor para lograr sus funciones correctamente y posee un tiempo reducido para realizar sus tareas, por ende se analiza que los trabajadores cumplen sus funciones activamente, con un nivel alto de motivación y aprendizaje activo. En definitiva, todos los grupos de trabajo de la institución de beneficencia poseen un alto nivel de control sobre la toma de decisiones relacionadas a su trabajo y a la organización del mismo, además los niveles de creatividad y el desarrollo de sus habilidades son altas.

$\mathrm{Al}$ incluir la variable apoyo social global, afecta positivamente en ambos géneros, ya que incrementa la habilidad para hacer frente a una situación de estrés, actuando el Apoyo Social como un amortiguador. Por lo cual los trabajadores en general, concuerdan que existe colaboración entre ellos y sus supervisores, por ende perciben que la dimensión demandas Laborales disminuye, es decir perciben que la cantidad de trabajo a realizar es menor, así mismo baja el nivel de presión con el tiempo para realizar sus tareas diarias, por lo que ayuda enormemente a la salud psicológica de los funcionarios, percibiendo un menor nivel de estrés.

En los grupos de trabajo calle, infanto adolescente, adulto mayor y educación inicial, se ubica en el cuadrante de" Baja Tensión", esto quiere decir que poseen alto nivel de control en sus actividades, y un bajo nivel de demandas psicológicas, por lo perciben un bajo nivel de presión al realizar sus actividades.

Para el grupo de trabajo de administración, si aumenta la dimensión demandas psicológicas y se mantiene un al alto control en las actividades realizadas, según el modelo de Karasek se traslada al cuadrante trabajo activo, este efecto disminuye la satisfacción laboral percibida.

En su mayoría, tanto en la muestra total como en los contratos a plazo fijo, la dimensión demandas psicológicas, tiene correlación negativa con las dimensiones de satisfacción laboral, esto quiere decir, que si la percepción de demandas psicológicas aumenta, disminuye la satisfacción laboral en trabajadores de la institución. Todas las dimensiones de satisfacción laboral en contratos indefinidos se correlacionan positivamente con las variables de control laboral y apoyo social global, esto quiere decir que si aumenta la percepción de estas variables, aumenta el nivel de satisfacción laboral en los trabajadores. Al aumentar la dimensión de apoyo social global la percepción de demandas psicológicas tiende a disminuir. Esta disminución de la demanda psicológica y el aumento del control laboral aumentan la satisfacción laboral.

Asimismo, en contratos a plazo fijo se observaron correlaciones estadísticamente significativas distintas en las dimensiones de inseguridad en el empleo y en la dimensión demandas psicológicas; ya que no hay correlaciones estadísticamente significativas respecto de las dimensiones de satisfacción.

En lo específico, si bien la muestra general arroja relaciones estadísticamente positivas entre las dimensiones de Estrés, Control Laboral y Apoyo Social Laboral Global con todas las dimensiones de Satisfacción por el trabajo, aparecen distinciones al segmentar la muestra en tipos de contrato indefinidos y contrato a plazo fijo. En la muestra en general se puede concluir que en la dimensión de demandas psicológicas posee relación negativa con la satisfacción en la forma en como realiza el trabajo y la satisfacción con la autonomía. Situación que se evidencia de manera marcada en el tipo de contrato a plazo fijo con correlaciones estadísticamente negativas para las dimensiones de satisfacción por el trabajo en general, satisfacción por el ambiente físico, satisfacción por la forma como realiza el trabajo, satisfacción con la oportunidad de desarrollo, satisfacción con la relación con jefe y satisfacción con la autonomía, este resultado que se da en el segmento a plazo fijo, sí está confirmando en la teoría, así se espera que se comporten estas variables. Para las dimensiones control laboral y apoyo social laboral global existe una relación estadísticamente positiva con todas las dimensiones de satisfacción laboral.

Luego dada las conclusiones presentadas, la hipótesis planteada en esta investigación que indica: "A mayor nivel de estrés laboral, menor nivel de satisfacción laboral", se puede inferir que está parcialmente aceptada. 


\section{REFERENCIAS}

1. Chiang $M$, Martin MJ, Núñez A. Relaciones entre el clima organizacional y la satisfacción laboral. Comillas: Universidad Pontificia de Comillas; 2010. 302 p. (Biblioteca Comillas Economí; 2)

2. Cavalcante J. Satisfacción en el trabajo de los directores de escuelas secundarias públicas de la región de Jacobina, Brasil [tesis] [en línea]. Barcelona: Universitat Autònoma de Barcelona; 2004 [citado sep 2018]. p. 108-118. Disponible en: http://es.slideshare.net/gracielacorvalan/jjcs1de1

3. Algag $R$, Brief $A$. Examination of alternative models of job satisfaction. Hum Relat. 1978; 31:91-98.

4. Snyder M, Ickes W. Personality and social behavior. In: Lindzey G, Aronson E, editors. Handbook of social psychology. 3rd ed. New York: Random House; 1985. p. 883-948.

5. Boada J, Tous J. Escalas de satisfacción laboral: una perspectiva dimensional. Rev Psicol: Universidad Tarraconensis. 1993; 15(2): 151-166.

6. Locke E. The nature and causes of job satisfaction. En: Dunnette M, editor. Handbook of industrial and organizational psychology. Chicago: Rand Mc Nally; 2009. p.1297-1349.

7. Mueller C, McCloskey J. Nurses' job satisfaction: A proposed measure. Nurs Res. 1990; 39: 113-117.

8. Newstrom J, Davis K. Organizational Behavior: Human Behavior at Work. New Delhi: McGraw-Hill; 2007.

9. Bravo M, Peiró J, Rodríguez I. Satisfacción laboral. En: Peiró J, Prieto $F$, editores. Tratado de psicologia del trabajo, 1. La actividad laboral en su contexto. España: Sintesis; 1996. p. 343-394.

10. Davis K, Newstrom J. Comportamiento humano en el trabajo. México: Mc Graw-Hill; 1993.

11. Alvarez J. Manual de Ergonomía y Psicología. Madrid: Mapfre; 2003.

12. Almodóvar $M$, Berjon $M$, Cuenca $M$, Fraile $A$, Garcia de Castro $M$, Del Hoyo M, Martin-Daza F, Nogareda C, Nogareda S, Oncins M, Urrutia M, Zubizarreta, I. Psicosociología del trabajo. Madrid: Instituto Nacional de Seguridad e Higiene en el Trabajo; 2003.

13. Llaneza F. Ergonomía y psicosociología aplicada; Manual para la formación del especialista. Valladolid: Lex Nova; 2009.

14. Bennis W. Desarrollo Organizacional: su naturaleza, sus orígenes y perspectivas. Bogotá: Fondo Educativo Interamericano; 1973.

15. Chandia F, Vidal $K$, Chiang M. Relación entre estrés laboral y adherencia a la Dieta Mediterránea (DM) en funcionarios de una institución de educación superior, Chile. Cienc Trab. 2016; 18(55):58-67.

16. Selye H. A syndrome produced by diverse nocuous agents. Nature. 1936:138: 32

17. Sandin B. El estrés: Un análisis basado en el papel de los factores sociales. Rev int psicol clín salud. 2003; 3(1): 141-157.

18. Lazarus R. Estrés y Emoción: Manejo e implicaciones en nuestra salud. Bilbao: Desclée de Brouwer; 2002.

19. OIT-OMS. Factores Psicosociales en el trabajo. Naturaleza, incidencia y prevención. Informe del Comité Mixto OIT/OMS sobre medicina del trabajo, 9a reunión; 18-24 sep 1984; Ginebra, Suiza. Ginebra: OIT; 1986. (Seguridad, Higiene y Medicina del Trabajo; 56).

20. Karasek R. Job demands, job decision latitude and mental strain: implications for job redesign. Admin Sci Quart. 1979; 24:285-308.

21. Johnson J, Hall E. Job strain, work place social support and cardiovascular disease: a cross-sectional study of a random sample of the Swedish working population. Am J Public Health. 1988; 78(10):1336-1342.

22. Karasek R, Theorell T. Healthy work: Stress, Productivity, and the reconstruction of Working Life. New York: Basic Books; 1990.

23. Mansilla F. Manual de riesgos psicosociales en el trabajo: Teoría y práctica; cómo influyen los factores de riesgos psicosociales en el trabajo y su prevención. Madrid: EAE; 2012. 260 p.

24. Cooper C, Baglioni A. A structural model approach toward the development of a theory of the link between stress and mental health. Brit J Med Psychol. 1988; 61(Pt 1): 87-102.

25. Kirk-Brown A, Wallace D. Predicting burnout and job satisfaction in workplace counselors: the influence of role stressors, job challenge, and organizational knowledge. J Employment Couns. 2004; 41(1): 29-37.

26. Sullivan S, Bhagat R. Organizational Stress, Job Satisfaction and Job Performance: Where Do We Go From Here? J Manage. 1992; 18(2): 353-374.

27. Beehr T, Newman J. Research on occupational stress: an unfinished enterprise. Pers psychol. 1998; 51(4): 835-844.

28. Judge T, Thoresen C, Bono J, Patton G. The job satisfaction-job performance relationship: A qualitative and quantitative review. Psychol Bull. 2001; 127(3): 376-407.

29. Jex S. Organizational Psychology: A Scientist-Practitioner Approach. New York: John Wiley \& Sons; 2002.

30. Rundmo T. Safety climate, attitudes and risk perception in Norsk Hydro. Safety Sci. 2000; 34(47):1-3.

31. Clarke N. Developing emotional intelligence abilities through team-based learning. Hum Resour Dev 0. 2010; 21(2):119-138.

32. Barthol R, Ku N. Regression under stress to first learned behavior. J Abnorm Soc Psych. 1959; 59(1):134-136.

33. Mandler G. The structure of value: Accounting for taste. In: Clark MS, Fiske ST, editors. Affect and Cognition; The 17th Annual Carnegie Symposium of Cognition. Hillsdale, NJ: Lawrence Erlbaum Associates; 1982. p. 3-36.

34. Hoffman D, Stetzer. A Cross-Level investigation of factors influencing unsafe behaviors and accidents. Pers psychol. 1996; 49(2): 307-339.

\section{Referencias complementarias:}

Cooper P, Taylor M, Cooper Z, Fairbum C. The development and validation of the body shape questionnaire. Int J Eat Disorder. 1987; 6(4): 485-494.

Peiró J. La Psicología del Trabajo y de las Organizaciones ante las transformaciones en el mundo del trabajo: Retos y perspectivas. En: Duarte $A_{\text {, }}$ Valentim J, organizadores. Psicología e Sociedade; Ciclo de Conferencias. Coimbra: Universidade de Coimbra; 1998. p.163-190. 